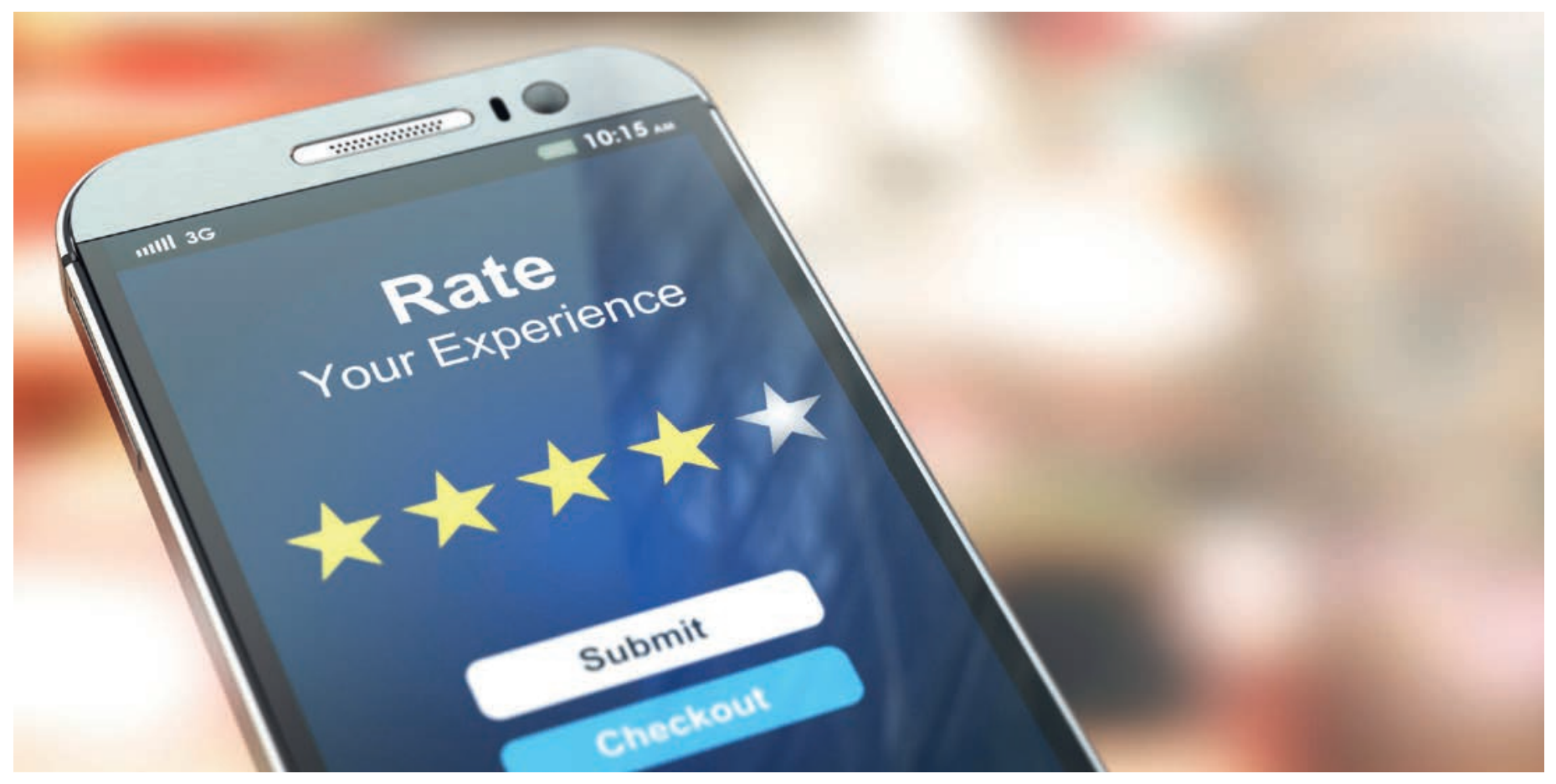

\title{
Rechtliche Aspekte von Bewertungen im Internet
}

\section{Michael Barnikol ${ }^{a}$, Reinhold Sojer ${ }^{b}$, Fabian Röthlisberger ${ }^{c}$}

${ }^{a}$ Dr. iur., Jurist Rechtsdienst, FMH; ${ }^{b}$ Dr. rer. biol. hum., Leiter Digitalisierung / eHealth, FMH;

${ }^{c}$ Wissenschaftlicher Mitarbeiter, Stv. Leiter Digitalisierung / eHealth, FMH

Infolge der erhöhten Popularität in anderen Branchen und verstärkten Fokus auf die Patientenzufriedenheit und Behandlungsqualität müssen sich Ärztinnen und Ärzte verstärkt mit dem Thema Online-Bewertungen auseinandersetzen. Inwiefern Online-Bewertungen im Gesundheitsbereich tatsächlich die Qualität der medizinischen Konsultation abbilden können, wird in der Literatur unterschiedlich diskutiert. Einerseits zeigen Studien, dass zwischen den Patientenbewertungen und objektiven medizinischen Outcome-Kriterien keine Korrelation besteht [1, 2]. Andererseits können

\section{Schweizer Patientinnen und Patienten nutzen Online-Bewertungsportale noch wenig.}

Patientinnen und Patienten sehr wohl Parameter wie die Infrastruktur, Organisation oder die Freundlichkeit bewerten, wobei diese Faktoren ebenso zu einer «guten Ärztin bzw. Arzt» beitragen [3]. Für die Ärzte- schaft entstehen bei nicht sachgerechten Online-Bewertungen grosse (rechtliche) Herausforderungen, insbesondere wegen berufsspezifischer Regulierungen wie z.B. der Unterstellung unter das Berufsgeheimnis [4-6]. Sie können sich gegenüber der Person, welche die Bewertung abgegeben hat, häufig nicht zur Wehr setzen, weil die Bewertungen oft anonym abgegeben werden. Auch die Möglichkeit, eine Gegendarstellung abzugeben, hilft meistens nicht weiter, da die Ärztin oder der Arzt hierfür oft Angaben zur Krankengeschichte machen müsste. Ohne Einwilligung des Patienten oder Entbindung vom Arztgeheimnis durch die kantonale Aufsichtsbehörde würde dies regelmässig einen Verstoss gegen das Arztgeheimnis bedeuten.

In der Schweiz bestehen mit Google und Websites wie medicosearch.ch, okdoc.ch, docapp.ch oder deindoktor.ch verschiedene Möglichkeiten zur Bewertung von Ärztinnen und Ärzten [7]. Jedoch nutzen Schweizer Patientinnen und Patienten diese Online-Bewertungs- 
portale noch wenig: Im Rahmen des eHealth-Barometers 2019 hat die FMH die Bevölkerung in einer Zusatzfrage nach der Nutzung von Ärztebewertungsportalen befragt. Dabei gaben nur $2 \%$ der Befragten an, ihre Ärztin oder ihren Arzt im letzten Jahr online bewertet zu haben. ${ }^{1}$ Fast drei Viertel der Befragten gaben zudem an, dass sie Online-Bewertungen bei der Arztwahl als nicht wichtig empfinden. Bei der Wahl einer Ärztin bzw. eines Arztes scheinen weiterhin persönliche Empfehlungen besser geeignet zu sein als unpersönliche öffentliche Informationen [8, 9]. Trotz der momentan geringen Nutzung ist davon auszugehen, dass die Anzahl von Online-Bewertungen aufgrund des Wunsches nach grösstmöglicher Transparenz in Zukunft zunehmen wird.

\section{Rechtliche Aspekte von Online- Bewertungsportalen}

Bewertungsportale umfassen meist zahlreiche Angaben zur Praxis und zur Person der Ärztin oder des Arztes. Aus juristischer Sicht steht daher die datenschutzrechtliche Beurteilung der Bewertungsportale im Vordergrund. Die einschlägige gesetzliche Regelung findet sich im Datenschutzgesetz (DSG). Dieses ist anwendbar, wenn Personendaten - d.h. alle Angaben, die sich auf eine bestimmte oder bestimmbare Person beziehen - bearbeitet werden (Art. 2 Abs. 1, Art. 3 lit. a, e und f DSG). Dazu zählt insbesondere die Veröffentlichung von Informationen und Bewertungen mit Bezug zu Ärztinnen und Ärzten im Internet. ${ }^{2}$

Somit müssen bei der Bewertung von Ärztinnen und Ärzten die allgemeinen Grundsätze für die Bearbeitung von Personendaten gemäss Art. 4 DSG und die Art. 12 ff. DSG beachtet werden. Insbesondere darf die Bearbeitung die Persönlichkeit der betroffenen Person nicht verletzen: Sie darf nicht entgegen Treu und Glauben erfolgen, unverhältnismässig sein, und es dürfen keine Daten gegen den ausdrücklichen Willen der betroffenen Person bearbeitet werden (Art. 12 Abs. 1 und Abs. 2 lit. a und b DSG i.V.m. Art. 4 DSG). Eine Persönlichkeitsverletzung kann darin bestehen, dass das Recht, über die eigenen Personendaten selbst zu bestimmten (sog. Recht auf informationelle Selbstbestimmung), oder andere Persönlichkeitsgüter ernsthaft bedroht werden. ${ }^{3}$

Dies ist z.B. der Fall, wenn die Bewertung gegen den ausdrücklichen Willen des betroffenen Arztes erfolgt, nachweislich falsche Tatsachenbehauptungen enthält oder einen ehrverletzenden bzw. herabwürdigenden Inhalt hat. ${ }^{4}$

Allerdings kann in bestimmten Fällen eine persönlichkeitsverletzende Datenbearbeitung ausnahmsweise gerechtfertigt sein. Eine solche Rechtfertigung kann durch Einwilligung des Verletzten, durch ein überwiegendes privates oder öffentliches Interesse oder durch ein Gesetz gegeben sein (Art. 13 Abs. 1 DSG). ${ }^{5}$

Es gibt in der Schweiz noch keine höchstrichterliche Rechtsprechung, die eine Orientierungshilfe für diese Interessenabwägung geben könnte.

Den betroffenen Rechten der Ärztin oder des Arztes in erster Linie das Recht auf informationelle Selbstbestimmung - stehen somit die wirtschaftlichen Interessen am Betrieb der Bewertungsplattform, das Interesse an einem freien Datenverkehr sowie die Meinungsfreiheit der Nutzer, die die Bewertungen abgeben, gegenüber. Es gibt in der Schweiz noch keine höchstrichterliche Rechtsprechung, die eine Orientierungshilfe für diese Interessenabwägung geben könnte. Der deutsche Bundesgerichtshof (BGH) hat sich in seiner Rechtsprechung zu besagter Interessenabwägung geäussert dies vor dem Hintergrund, dass nach $§ 29$ Abs. 1 S. 1 Nr. 1 des deutschen Bundesdatenschutzgesetzes die Erhebung und Speicherung personenbezogener Daten zum Zweck der Übermittlung nur dann zulässig ist, wenn kein Grund zu der Annahme besteht, dass der Betroffene ein schutzwürdiges Interesse an dem Ausschluss der Erhebung oder Speicherung hat. ${ }^{6}$

In besagtem Fall klagte eine Dermatologin vor dem BGH, da die Bewertungsplattform Jameda auf ihrem Online-Profil Werbung für konkurrierende Ärztinnen und Ärzte einblendete. Der BGH stellte fest, dass die Bewertungsplattform ihre Stellung als neutrale Informationsmittlerin verlässt und damit für die Ärztin ein "schutzwürdiges Interesse an dem Ausschluss der Speicherung ihrer Daten» besteht.

An der Verbreitung von beleidigenden oder nachweisbar falschen Bewertungen besteht jedenfalls kein überwiegendes Interesse. Dementsprechend verbieten Portalbetreiber in ihren Nutzungsbedingungen regelmässig solche Aussagen und bieten die Möglichkeit an, ihnen diese zu melden, damit sie gelöscht werden können. Dies ist sinnvoll, da auf diese Weise ein langwieriger und teurer Rechtsstreit im Vorfeld vermieden werden kann. Es wäre jedoch wünschenswert und im Sinne des Rechts auf informationelle Selbstbestimmung, wenn diejenigen Ärzte, die eine Aufnahme in einem Bewertungsportal ablehnen, jederzeit die Möglichkeit hätten, ihren Brancheneintrag (inklusive negativer, aber auch positiver Bewertungen und anderer Personendaten) insgesamt zu löschen. Dies würde das Geschäftsmodell der Bewertungsportale nicht in Frage stellen und die - nach Art. 12 Abs. 2 lit b jedenfalls dem Grundsatz nach unzulässige - Bearbeitung von Daten 
gegen den Willen der betroffenen Ärzte in einer nicht unerheblichen Anzahl von Fällen vermeiden.

Wenn eine rechtswidrige Persönlichkeitsverletzung gegeben ist, können die betroffenen Ärztinnen und Ärzte vom Portalbetreiber die Unterlassung der Datenbearbeitung und die Vernichtung oder Berichtigung der Daten verlangen (Art. 15 Abs. 1 DSG i.V.m. Art. 28, 28a und $28 \mathrm{l} \mathrm{ZGB).} \mathrm{Diese} \mathrm{Ansprüche} \mathrm{richten} \mathrm{sich} \mathrm{gegen}$ sämtliche Personen, die an der widerrechtlichen Persönlichkeitsverletzung mitgewirkt haben.?

Darüber hinaus enthält das DSG einen allgemeinen und umfassenden Anspruch auf Auskunft gegenüber Inhabern von Datensammlungen ${ }^{8}$ (Art. 8 Abs. 1 DSG). Der Begriff des «Inhabers» ist sehr weit gefasst: Darunter fällt jeder, der den Zweck der Sammlung festlegt und die Bearbeitungsmittel und -methoden bestimmt sowie über deren Inhalt, also über die Existenz und die wesentliche Ausgestaltung, entscheidet (Art. 3 Bst. i DSG). Es ist davon auszugehen, dass die Betreiber der Bewertungsplattformen als Inhaber von Datensammlungen anzusehen sind ${ }^{9}$ und die betroffenen Ärztinnen und Ärzte daher einen Auskunftsanspruch gegen den Portalbetreiber haben.

\section{Empfehlungen der FMH zu Online- Bewertungen}

7 Rosenthal/Jöhri, a.a.O., Art. 12 Rz. 10

8 Eine Datensammlung ist jeder Bestand von Personendaten, der so aufgebaut ist, dass die Daten nach betroffenen Personen erschliessbar sind (Art. 3 Bst. g DSG)

9 Ebenso: Erläuterungen des EDÖB zu Bewertungsplattformen im Internet, a.a.O.

Korrespondenz:

FMH

Abteilung Rechtsdienst Elfenstrasse 18, Postfach 300 CH-3000 Bern 15

lex[at]fmh.ch
Da Online-Bewertungen gravierende Auswirkungen auf die einzelne Berufsperson und deren Arztpraxis haben können, erarbeitet die FMH für ihre Mitglieder aktuell verschiedene Hilfestellungen. Neben den hier publizierten rechtlichen Aspekten von Online-Bewertungsportalen wird aktuell ein Kommunikationsleitfaden für den Umgang mit Online-Bewertungen erarbeitet. Zudem werden Qualitätskriterien definiert, welche aus Sicht der FMH bei einem zuverlässigen Bewertungsportal erfüllt sein müssen. Die Qualitätskriterien sind als Forderung an die Portalbetreiber zu verstehen und sind Basis einer fairen Bewertung von Ärztinnen und Ärzten. Die Resultate dieser laufenden Arbeiten werden im Sommer 2019 in geeigneter Form publiziert.
Fazit

- Online-Bewertungen von Ärztinnen und Ärzten werden infolge des Wunsches nach grösstmöglicher Transparenz zunehmen.

- Die Veröffentlichung von Daten von Ärztinnen und Ärzten ist die Bearbeitung von Personendaten mit der Folge, dass das Datenschutzgesetz (DSG) anwendbar ist.

- Bei Einträgen, welche persönlichkeitsverletzend sind, hat die Ärztin oder der Arzt Anspruch auf Vernichtung oder Berichtigung der Daten.

- In Bezug auf Online-Bewertungen von Ärztinnen und Ärzten gibt es in der Schweiz noch keine gefestigte Rechtsprechung.

- Die FMH erarbeitet für ihre Mitglieder Empfehlungen im Sinne eines Kommunikationsleitfadens. Zudem werden Qualitätskriterien von Bewertungsportalen definiert, welche gerichtet an die Portalbetreiber eine faire Bewertung von Ärztinnen und Ärzten einfordern.

\section{Bildnachweis}

(c) Maksym Yemelyanov | Dreamstime.com (Symbolbild)

\section{Literatur}

1 Okike K, Peter-Bibb TK, Xie KC, Okike ON. Association between physician online rating and quality of care. J Med Internet Res. 2016.

2 Daskivich TJ, Houman J, Fuller G, Black JT, Kim HL, Spiegel B. Online physician ratings fail to predict actual performance on measures of quality, value, and peer review. J Am Med Informatics Assoc. 2018.

3 Rothenfluh F, Schulz PJ. Physician rating websites: What Aspects are important to identify a good doctor, and are patients capable of assessing them? A mixed-methods approach including physicians' and health care consumers' perspectives. J Med Internet Res. 2017.

4 Meienberg O. Arzt-Entwertung im Internet. Schweiz Ärzteztg. 2018

5 Meienberg O. Praxisärzte-Rating auf Abwegen. Arzt-Entwertung im Internet. Schweiz Ärzteztg. 2018.

6 Mahler D. FMH sollte sich der Problematik «Ärztebewertungen im Internet» dringlich annehmen! Schweiz Ärzteztg. 2018

7 Büchi S. Arztbewertung - Wie viele Sterne dürfen's denn sein. SRF [Internet]. 2016; Available from: https://www.srf.ch/sendungen/ puls/gesundheitswesen/arztbewertung-wie-viele-sterne-duerfens-denn-sein

8 McLennan S, Strech D, Meyer A, Kahrass H. Public awareness and use of German physician ratings websites: Cross-sectional survey of four North German cities. Journal of Medical Internet Research. 2017.

9 Burkle CM, Keegan MT. Popularity of internet physician rating sites and their apparent influence on patients' choices of physicians. BMC Health Serv Res. 2015. 